\title{
El capital intelectual y el capital emprendedor como impulsores del crecimiento económico regional
}

\author{
Luis Lupiáñez Carrillo ${ }^{a}$ Cristina López-Cózar Navarro ${ }^{\mathrm{b}, *}$ y Tiziana Priede Bergamini ${ }^{\mathrm{c}}$ \\ a Organismo para el Desarrollo Socioeconómico, Ayuntamiento de Baracaldo, Baracaldo, Vizcaya, España \\ b Departamento de Economía Agraria, Estadística y Organización de Empresas, Universidad Politécnica de Madrid, Madrid, España \\ ' Departamento de Empresa, Universidad Europea de Madrid, Madrid, España
}

Recibido el 6 de junio de 2016; aceptado el 27 de septiembre de 2016

Disponible en Internet el 27 de octubre de 2016

\section{CÓDIGOS JEL \\ M13; \\ L26; \\ 018; \\ 01}

\section{PALABRAS CLAVE \\ Emprendimiento; Crecimiento económico; Innovación; Conocimiento; España}

\section{JEL CLASSIFICATION M13; \\ L26; \\ 018; \\ 01}

\section{KEYWORDS}

Entrepreneurship; Economic growth;
Resumen Existe un amplio consenso en aceptar una relación directa entre la capacidad de creación de empresas de un país y su crecimiento económico. Sin embargo, resulta interesante profundizar en el análisis de los factores que incrementan el crecimiento económico regional. Por tanto, este trabajo tiene como objetivo comprobar si el capital emprendedor es el elemento catalizador que, en combinación con un alto nivel de capital intelectual, se convierte en un factor de crecimiento económico de las comunidades autónomas españolas, en el contexto de la economía del conocimiento. Las conclusiones del estudio apuntan a la necesidad de continuar con una labor de difusión y potenciación de la cultura emprendedora en el ámbito de la economía del conocimiento, especialmente entre los microempresarios y los pequeños empresarios, que son los que más contribuyen a la actividad económica y a la productividad laboral.

(c) 2016 Asociación Cuadernos de Economía. Publicado por Elsevier España, S.L.U. Todos los derechos reservados.

Intellectual capital and entrepreneurial capital as driver forces of regional growth

Abstract There is broad consensus accepting a direct relationship between the ability to create companies in a country and its economic growth. However, it is interesting to go into greater depth in the analysis of the factors that increase the economic growth of the regions. This study aims to demonstrate whether entrepreneurship is the catalyst element, which in combination with a high level of intellectual capital becomes a factor for economic growth in the Spanish autonomous regions, within the context of the knowledge economy. The findings of the study highlight the need to disseminate and promote the entrepreneurial culture in the

\footnotetext{
* Autor para correspondencia.

Correo electrónico: cristina.lopezcozar@upm.es (C. López-Cózar Navarro).
} 
Innovation;

Knowledge;

Spain field of knowledge economy, especially among micro- and small entrepreneurs, who are major contributors to economic activity and labour productivity.

(c) 2016 Asociación Cuadernos de Economía. Published by Elsevier España, S.L.U. All rights reserved.

\section{Introducción}

Es ampliamente reconocido que existe una relación directa entre la capacidad de creación de empresas de un país y su crecimiento económico. Schumpeter (1934) ya apuntó que la causa fundamental del crecimiento económico era la innovación a través de la puesta en marcha de iniciativas empresariales, las cuales actúan diseminando el conocimiento mediante el aprovechamiento de las oportunidades emprendedoras, mejorando la competitividad a través del incremento del número de empresas y diversificando la estructura empresarial (Audretsch y Keilbach, 2004a). Posteriormente, distintos estudios han confirmado que la creación de empresas constituye un factor clave para lograr una mejora en la productividad (Audretsch, 2009), así como que representa el motor de las economías en los países desarrollados (García et al., 2010; Aubry et al., 2015; Huggins y Thompson, 2015).

Por su parte, y a consecuencia de la globalización económica y de la universalización de las tecnologías de la información y la comunicación, se ha desarrollado un nuevo paradigma social denominado «sociedad de la información». Esta ha dado lugar a un nuevo modelo económico: la economía del conocimiento, en la que la innovación aparece como el factor de competitividad fundamental y los recursos intangibles se constituyen como los elementos capaces de generarla. Así, la plena entrada en la denominada sociedad del conocimiento (Bueno, 1998; Delgado-Verde et al., 2011) implica que las regiones pasan a ser los ámbitos básicos de referencia para medir el desarrollo (Malhotra, 1998). Así, la innovación, el conocimiento, el aprendizaje, su producción y su crecimiento, que son la base de la sociedad del conocimiento, son a la vez el resultado de un proceso complejo de relaciones e interdependencias entre diferentes agentes locales, dentro de los denominados sistemas regionales de innovación. Ante este nuevo paradigma, todos los agentes económicos -y por supuesto los diversos territoriosdeben adaptar sus economías y redefinir, dentro de este nuevo modelo, qué factores los hacen más competitivos.

El conocimiento se perfila como el principal recurso intangible que poseen las regiones para mantener una ventaja competitiva, y la creación de empresas como el instrumento fundamental para conducir a una economía hacia este nuevo modelo. No obstante, como ya señaló Galbraith en 1969, del carácter estático del conocimiento es preciso avanzar hacia un concepto más dinámico que facilite el desarrollo de un proceso ideológico más completo denominado capital intelectual (IC) (Bontis, 1998); así, el IC se refiere al conjunto de recursos intangibles y capacidades relacionadas con el conocimiento que permiten alcanzar una ventaja competitiva sostenible (Edvinsson y Sullivan, 1996). Por su parte, respecto a la creación de empresas, la capacidad de una región para generar actividad empresarial se conoce como capital emprendedor (E) (Romer, 1986).

Es importante señalar que un elevado nivel de IC y/o E no implica necesariamente niveles elevados de bienestar, pero niveles bajos de IC y/o E sí suponen niveles bajos de bienestar. Por ello, resulta interesante profundizar en el conocimiento existente de ambos factores y sus consecuencias sobre el crecimiento y el bienestar en el ámbito regional. Efectivamente, este emprendimiento de carácter territorial despierta un amplio interés en la literatura académica, ya que representa una parte importante de la actividad económica local, por lo que es preciso seguir avanzando en el desarrollo de estos temas (Müller, 2016). Así pues, el principal objetivo del presente trabajo es el estudio de las relaciones entre el IC, el capital emprendedor y la actividad económica, con el fin de responder a la siguiente pregunta: ¿es suficiente la combinación existente de IC y capital emprendedor para generar crecimiento económico en el ámbito regional?

Con este objetivo, el presente artículo pretende establecer el impacto de la acumulación de IC y E en el contexto regional, pues siguiendo a Audretsch (2007), se considera que es el mejor nivel para estudiar el emprendimiento. Así, el ámbito geográfico elegido es el de las 17 comunidades autónomas (CCAA) españolas, para lo cual se ha construido un panel de datos de dichas comunidades. El estudio se realiza para el periodo 2000-2008 con el fin de no incorporar en el análisis los años de crisis económica y no distorsionar así los resultados obtenidos.

Este trabajo contribuye a mejorar la comprensión de las relaciones causales de dependencia e interdependencia entre IC y E, con respecto a la actividad económica y la productividad desde una perspectiva regional. Por otra parte, también aporta un interesante avance en la definición del concepto conocimiento, pues aunque ha sido ampliamente estudiado en la literatura existente, no ha sido posible hasta la fecha establecer estándares de utilización universal en su aplicación empírica; en este sentido, el presente trabajo introduce un cambio con respecto al modelo original al sustituir los factores utilizados anteriormente por el IC. No obstante, la principal aportación que se presenta en este artículo es la contrastación empírica de que el capital emprendedor se puede considerar el elemento catalizador que, en combinación con un alto nivel de IC, se convierte en un factor de crecimiento económico regional. 
Para conseguir el objetivo propuesto, el trabajo se estructura del siguiente modo: en el siguiente epígrafe se establece el marco teórico, revisando los conceptos analizados en el modelo y las relaciones entre ellos, justificando así las hipótesis planteadas. A continuación se describe la metodología empleada, la medición de las variables y se comentan los resultados obtenidos. Finalmente, se exponen las principales conclusiones, las limitaciones del estudio y las futuras líneas de investigación.

\section{Marco teórico e hipótesis}

\subsection{El conocimiento y el capital intelectual}

El conocimiento es un concepto que ha sido objeto de numerosos estudios, y sin embargo aún sigue resultando un término amplio y difuso, lo que implica una importante dificultad para definirlo de forma concreta y comprenderlo. Así, por ejemplo, Davenport y Prusak (1997) lo definen como una mezcla de información y saber hacer que son útiles para la acción; Muñoz-Seca y Riverola (1997) lo consideran como la capacidad de resolver un determinado conjunto de problemas con una efectividad determinada, y Stewart (1997) lo define como la información que posee valor para una organización y que le brinda operatividad en su competencia por satisfacer las demandas del mercado. De todas estas definiciones, cabe destacar la existencia de un aspecto endógeno del concepto, que adquiere valor y significado con relación al contexto en el que este se desarrolla. Así, la importancia del conocimiento está fuertemente ligada con el valor que se le atribuye y los resultados que se esperan de él, por lo que, desde el punto de vista de las organizaciones, el conocimiento es la información que permite generar acciones asociadas a satisfacer las demandas del mercado (Porter y Millar, 1986).

Esta declaración del conocimiento como un activo valioso para las organizaciones dio lugar a un incremento del interés sobre cómo gestionarlo (Zack, 2002; López-Nicolás y Meroño-Cerdán, 2011). La diversidad de enfoques hace que sea difícil establecer una única definición de gestión del conocimiento, si bien una de las que cuenta con mayor aceptación es la propuesta por Nonaka y Takeuchi (1995), quienes consideran que es la capacidad que la organización tiene para crear conocimiento nuevo, diseminarlo e incorporarlo en productos, servicios y sistemas. Así, se puede afirmar que la gestión del conocimiento es un proceso integral que abarca el aprendizaje de la organización y la gestión del IC en estrecha relación con la información, el conocimiento y el aprendizaje, y las actividades relacionadas con su creación, organización y explotación (Bueno, 1998; Davenport y Prusak, 1998; Skyrme, 2001). Por lo tanto, se considera que la gestión del conocimiento es la base para el desarrollo de otras capacidades y, en consecuencia, es la capacidad dinámica más importante de la empresa (Subramaniam y Youndt, 2005; Martín de Castro et al., 2009). En este sentido, Stewart (1997) afirma que la gestión del conocimiento es el conjunto de procesos que hacen que el IC de la empresa crezca.

A pesar de no existir una definición consensuada, diversos autores coinciden en señalar que IC se refiere al conjunto de recursos intangibles y capacidades relacionadas con el conocimiento que permiten a la organización alcanzar una ventaja competitiva sostenible (Edvinsson y Sullivan, 1996; Stewart, 1997; Bontis, 1998; Sullivan, 1998; Edvinsson y Malone, 1999; Subramaniam y Youndt, 2005; Kristandl y Bontis, 2007; Viedma, 2007; Simó y Sallán, 2008; Delgado-Verde et al., 2011). Más recientemente, Barrena-Martínez et al. (2016) consideran que IC es una combinación de intangibles, tales como conocimientos, experiencias o destrezas capaces de generar una ventaja diferencial en el mercado. Asimismo, López y Nevado (2016) apuntan que se trata de conjunto de activos relacionados con el capital humano y con otros estructurales (capacidad de innovación, relaciones con los clientes, calidad de los procesos, capital cultural, etc.) que permiten a la empresa aprovechar mejor las oportunidades.

Desde la década de los noventa, son muchos los esfuerzos que se han realizado, tanto en el ámbito académico como en el empresarial, para la medición y gestión del IC. En este sentido, Castilla-Polo y Gallardo-Vázquez (2016) realizan una revisión profunda de la literatura al respecto y un análisis exhaustivo sobre la importancia de los intangibles en la economía actual. En general, los diferentes modelos planteados comparten una estructura común, si bien difieren tanto en las metodologías que los desarrollan como en el tipo de datos que los alimentan, lo que muestra que no existe un modelo universal que pueda ser aplicado de forma generalizada, sino que cada situación necesita un modelo adaptado a ella.

En el contexto actual, donde la internacionalización de la producción es habitual, así como el aumento de las actividades de servicios e intercambio entre las mismas, las regiones son el principal escenario de desarrollo, pues es precisamente en ellas donde se deciden las principales inversiones y las localizaciones industriales y de servicios. En este sentido, Bradley (2003) considera que el IC permite transformar el conocimiento y otros recursos intangibles en recursos generadores de riqueza, no solo para las organizaciones, sino también para las naciones. Si bien, como señalan Acs et al. (2013), la capacidad de transformar el conocimiento en conocimiento económico implica no solo un conjunto de habilidades, sino también la proximidad a la fuente del mismo. Así, los territorios que desarrollen actividades basadas en el conocimiento serán los que alcancen una mayor riqueza en el futuro (Ramírez, 2010). El gran potencial de desarrollo de las regiones hace posible dar respuesta a los desafíos generados por el incremento de la competitividad, creando vínculos entre los diferentes procesos de ajuste productivo y organizativo, y el uso de recursos propios, así como a la propagación de las innovaciones y el fortalecimiento de los lazos con otras regiones (Malhotra, 2000). Así, los diversos territorios de un país son algo más que un lugar físico en el mapa; lo importante no es tanto su delimitación o ubicación geográfica, sino que se basan en un sistema de interacción de actores, conocimientos, bienes y servicios, de acuerdo a reglas concretas de funcionamiento (Veciana, 2007).

Ante este nuevo enfoque, los agentes económicos, y por supuesto las regiones, deben adaptar sus economías y redefinir qué factores los hacen más competitivos. En el presente trabajo se pretende estudiar el impacto de la acumulación de IC en las CCAA españolas, con el fin de conocer en qué medida puede ser un factor impulsor de crecimiento. Con este propósito, las hipótesis planteadas son las 
siguientes: $\mathrm{H}_{1} \mathrm{~A}$ mayor intensidad de IC en la región, mayor será el nivel de actividad económica. $\mathrm{H}_{2} \mathrm{~A}$ mayor intensidad de IC en la región, mayor será la productividad laboral.

\subsection{El capital emprendedor}

Se puede definir E como la capacidad de un territorio para crear nuevas empresas (Romer, 1986; Audretsch y Keilbach, 2004a, 2004b, 2005), incluyendo diversos aspectos legales, institucionales y sociales (Audretsch, 2009). Actualmente, los países y las regiones concentran sus esfuerzos en fomentar el $E$ buscando la incorporación de nuevos empresarios a la actividad económica (Bosma, 2009). La literatura previa confirma que la creación de nuevas empresas contribuye al crecimiento económico, a impulsar la innovación y a mejorar la competitividad y, en consecuencia, a lograr un mayor bienestar para la sociedad (Acs y Szerb, 2007; Audretsch, 2007, 2009; García et al., 2010; Benito-Hernández et al., 2012; Galindo y Méndez, 2014, Castaño et al., 2016).

Tanto Audretsch y Keilbach (2004a) como Carree y Thurik (2006) probaron que el incremento de la actividad emprendedora está relacionado con altas tasas de crecimiento económico, y también encontraron que elevadas tasas de creación de empresas se asocian con altas tasas de crecimiento y bajas tasas de desempleo. En la misma línea, diversos estudios empíricos, como los de Callejón y Segarra (1999) en España, Reynolds (1999) en Estados Unidos, Fölster (2000) en Suecia, Audretsch y Fritsch (2002) en Alemania, Piergiovanni et al. (2012) en Italia, Matejovsky et al. (2014) en Canadá o Aubry et al. (2015) en Francia, entre otros, confirman que cuanto mayor sea la tasa de creación de empresas de un país, mayor será su nivel de crecimiento económico.

Por su parte, generalmente se ha considerado que la unidad de medida del E es la región o la ciudad, puesto que permite aprovechar las externalidades del conocimiento que tienden a agruparse dentro de una zona geográficamente acotada (Audretsch y Feldman, 1996). Acs y Armington (2004) llevaron a cabo una investigación en Estados Unidos, confirmando que aquellas regiones en las que existían spillovers fruto de la concentración de conocimiento, junto con una mayor tasa de actividad emprendedora, mostraban una tasa de crecimiento más elevada.

No obstante, no todos los emprendedores tienen un carácter homogéneo. La literatura sobre el espíritu empresarial sugiere que la identificación del tipo de emprendimiento es esencial para establecer la relación entre la actividad emprendedora y el crecimiento económico. Diversos tipos de iniciativa empresarial pueden tener diferentes impactos en el crecimiento económico de una región (Sternberg y Wennekers, 2005). Audretsch y Keilbach (2004a) encuentran cierta evidencia que sugiere que la actividad emprendedora en el ámbito del I+D puede tener un mayor impacto a largo plazo que en otros tipos de empresas. Así, además de la evidencia de relación positiva entre el capital emprendedor y el crecimiento económico, existen otras experiencias para las que los resultados son dispares en función de variables como el factor desencadenante de la puesta en marcha del proyecto empresarial (Acs et al., 2004; Bosma, 2009) o en función del nivel de desarrollo (Wennekers, 2006; Acs y Szerb, 2007).

Por lo tanto, con el objetivo de comprobar si existe una relación positiva entre IC, E y el crecimiento económico en el contexto de la economía del conocimiento de las CCAA españolas, se plantean las siguientes hipótesis: $\mathrm{H}_{3} \mathrm{E}$ ejerce un impacto positivo en el nivel de actividad económica en el ámbito de la economía del conocimiento. $\mathrm{H}_{4} \mathrm{E}$ ejerce un impacto positivo en la productividad laboral en el ámbito de la economía del conocimiento.

Además del indicador global $E$, se han definido otros 9 indicadores, de acuerdo con el número de empleados de las empresas, con el fin de determinar su tamaño: E sin asalariados, E de 1 a 2 empleados, E de 3 a 5 empleados, E de 6 a 9 empleados, E de 10 a 19 empleados, E de 20 a 49 empleados y E de 50 a 99 empleados. Esta clasificación incluye el $99,60 \%$ de las empresas como promedio en el periodo estudiado. Asimismo, se establecen 2 indicadores fruto de la agrupación de alguno de los anteriores, como son: un indicador que agrupa a las empresas $E$ entre 3 y 9 , y un segundo que contempla el rango $E$ entre 3 y 19 empleados.

Esta segmentación responde a los criterios de tamaño inicial de la empresa y a la necesidad de establecer un límite a lo que se puede considerar como iniciativa emprendedora, y todo ello en el contexto de las actividades que define el escenario planteado por Sánchez y Arellano (2012), en cuanto a actividades relacionadas con la economía del conocimiento, tales como las consideradas de alta intensidad tecnológica, ciertos servicios y otros sectores creativos.

Para recoger la segmentación propuesta en función del tamaño de las empresas creadas y obtener con ello más información, se plantean las siguientes subhipótesis: $\mathrm{H}_{3 \mathrm{~A}} \mathrm{E}$ de empresas sin asalariados ejerce un impacto positivo en el nivel de actividad económica en el ámbito de la economía del conocimiento. $\mathrm{H}_{3 \mathrm{~B}} \mathrm{E}$ de empresas de 1 a 2 empleados ejerce un impacto positivo en el nivel de actividad económica en el ámbito de la economía del conocimiento. $\mathrm{H}_{3 \mathrm{C}} \mathrm{E}$ de empresas de 3 a 5 empleados ejerce un impacto positivo en el nivel de actividad económica en el ámbito de la economía del conocimiento. $\mathrm{H}_{3 \mathrm{D}} \mathrm{E}$ de empresas de 6 a 9 empleados ejerce un impacto positivo en el nivel de actividad económica en el ámbito de la economía del conocimiento. $\mathrm{H}_{3 \mathrm{E}} \mathrm{E}$ de empresas de 3 a 9 empleados ejerce un impacto positivo en el nivel de actividad económica en el ámbito de la economía del conocimiento. $\mathrm{H}_{3 \mathrm{~F}} \mathrm{E}$ de empresas de 10 a 19 empleados ejerce un impacto positivo en el nivel de actividad económica en el ámbito de la economía del conocimiento. $\mathrm{H}_{3 \mathrm{G}} \mathrm{E}$ de empresas de 3 a 19 empleados ejerce un impacto positivo en el nivel de actividad económica en el ámbito de la economía del conocimiento. $\mathrm{H}_{3 \mathrm{H}} \mathrm{E}$ de empresas de 20 a 49 empleados ejerce un impacto positivo en el nivel de actividad económica en el ámbito de la economía del conocimiento. $\mathrm{H}_{31} \mathrm{E}$ de empresas de 50 a 99 empleados ejerce un impacto positivo en el nivel de actividad económica en el ámbito de la economía del conocimiento.

Y a su vez, en relación con la productividad laboral, se plantean las siguientes: $\mathrm{H}_{4 \mathrm{~A}} \mathrm{E}$ de empresas sin asalariados ejerce un impacto positivo en la productividad laboral en el ámbito de la economía del conocimiento. $\mathrm{H}_{4 \mathrm{~B}} \mathrm{E}$ de empresas de 1 a 2 empleados ejerce un impacto positivo en la productividad laboral en el ámbito de la economía del conocimiento. $\mathrm{H}_{4 \mathrm{C}} \mathrm{E}$ de empresas de 3 a 5 empleados ejerce un impacto positivo en la productividad laboral en el ámbito de la economía del conocimiento. $\mathrm{H}_{4 \mathrm{D}} \mathrm{E}$ de empresas de 6 a 9 empleados ejerce un impacto positivo en la productividad laboral en el ámbito de la economía del conocimiento. $\mathrm{H}_{4 \mathrm{E}} \mathrm{E}$ 
de empresas de 3 a 9 empleados ejerce un impacto positivo en la productividad laboral en el ámbito de la economía del conocimiento. $\mathrm{H}_{4 \mathrm{~F}} \mathrm{E}$ de empresas de 10 a 19 empleados ejerce un impacto positivo en la productividad laboral en el ámbito de la economía del conocimiento. $\mathrm{H}_{4 \mathrm{G}} \mathrm{E}$ de empresas de 3 a 19 empleados ejerce un impacto positivo en la productividad laboral en el ámbito de la economía del conocimiento. $\mathrm{H}_{4 \mathrm{H}} \mathrm{E}$ de empresas de 20 a 49 empleados ejerce un impacto positivo en la productividad laboral en el ámbito de la economía del conocimiento. $\mathrm{H}_{4 \mid} \mathrm{E}$ de empresas de 50 a 99 empleados ejerce un impacto positivo en la productividad laboral en el ámbito de la economía del conocimiento.

\section{Metodología}

Desde un punto de vista teórico, la propuesta planteada trasciende el modelo neoclásico de Solow (1956), que fue el primer modelo de crecimiento, en el cual la producción económica estaba generada por la interacción del capital físico y del trabajo, y su crecimiento, por el cambio tecnológico, que era de carácter exógeno. Nuestra propuesta se extiende más allá de la consideración de estas 2 variables como los factores de crecimiento económico de las regiones, ampliando la función de producción con 2 nuevas variables que se detallan a continuación. Asimismo, proponemos no aceptar ciertos supuestos básicos del modelo, como los rendimientos constantes a escala y la productividad de los factores decrecientes, sino considerar la posibilidad de rendimientos de escala crecientes y los efectos de propagación.

En este contexto, la presente investigación encuentra en la teoría del crecimiento endógeno de Romer (1986, 1990) un marco adecuado, incorporando a la función de producción factores intangibles, los cuales resuelven el carácter exógeno que le atribuían los neoclásicos al progreso tecnológico, y que además se convierte en la causa de generación de una productividad creciente de los factores, debido a los spillovers generados por estos nuevos factores. En nuestro caso, IC y E se convierten en esos 2 nuevos factores intangibles que junto con los 2 factores neoclásicos, capital y trabajo, serán incorporados en una función de producción Cobb-Douglas (1928), tal y como se planteaba en el modelo original neoclásico, si bien adoptando los supuestos básicos del modelo de crecimiento endógeno. Esta propuesta metodológica ya fue utilizada antes por Audretsch y Keilbach (2004a, b, 2005).

Así, el modelo permite establecer un vínculo entre el $\mathrm{E}$ y el crecimiento económico, convirtiéndolo en un canal de difusión de conocimientos. En este sentido, Acs et al. (2004) apuntan la existencia de un filtro entre el conocimiento y el conocimiento económicamente útil, y señalan al $\mathrm{E}$ como un mecanismo que facilita la utilización del conocimiento para el crecimiento económico, a través de la creación de nuevas empresas.

Con respecto a la variable que representa el factor conocimiento, son habitualmente utilizados como indicador conocimiento (R) de Romer (1986) o (H) capital humano de Lucas (1988), el número de trabajadores en el ámbito del I+D o la inversión en este concepto en función del PIB (Griliches, 1979; Jaffe, 1989; Audretsch y Feldman, 1996; Audretsch y Keilbach, 2004b). Una aportación del presente trabajo con respecto al modelo original consiste en sustituir los factores citados por IC.
Tabla 1 Variables del modelo propuesto

\begin{tabular}{lll}
\hline Tipo de variable & Variable & Nombre \\
\hline Dependiente & Nivel de producción & $\mathrm{Y}$ \\
& Productividad laboral & $\mathrm{Y} / \mathrm{L}$ \\
Independiente & Capital físico & $\mathrm{K}$ \\
& Trabajo & $\mathrm{L}$ \\
& Intensidad de capital & $\mathrm{K} / \mathrm{L}$ \\
& Capital emprendedor & $\mathrm{E}$ \\
& Capital intelectual & $\mathrm{IC}$ \\
\hline
\end{tabular}

\subsection{Muestra y procedencia de los datos}

Los datos utilizados para la realización de este estudio se han obtenido de 2 fuentes de carácter oficial: el Instituto Nacional de Estadística (INE) y Eurostat. Se han considerado como unidades de estudio las 17 CCAA españolas, eliminando del análisis las 2 ciudades autónomas de Ceuta y Melilla, ante la carencia o la falta de desagregación de los datos de estas 2 ciudades.

Con respecto al ámbito temporal, se ha establecido el periodo 2000-2008, para no incorporar en el estudio los años de crisis económica con el fin de no distorsionar los resultados. Se trata, no obstante, de un periodo de tiempo que se considera suficiente para observar el impacto de las 2 variables de estudio, en un contexto económico y político-social homogéneo (tabla 1).

\subsection{Variables utilizadas}

\subsubsection{Variables dependientes}

Nivel de producción (Y). La producción se mide como el valor agregado bruto a precios constantes de cada una de las CCAA en cada periodo, expresada en miles de euros. Se trata de un indicador del nivel de actividad económica por el lado de la oferta, y representa la suma de los gastos de sueldos y salarios, los pagos a las dotaciones de capital, los intereses, las utilidades, los impuestos y el excedente bruto de explotación. Estos datos fueron obtenidos de Eurostat, a través de sus estadísticas regionales, clasificación NUTS 2.

Productividad laboral (Y/L). Es una variable derivada de la relación entre el nivel de producción (Y) y la población económicamente activa en la comunidad autónoma (L); representa la renta generada por cada trabajador activo, actúa como una medida de la competitividad de las CCAA y se expresa en euros por trabajador activo.

\subsubsection{Variables independientes}

Capital físico (K). El stock de capital, definido como el conjunto de estructuras y equipos utilizados en la producción (Sheram y Soubbotina, 2005), se ha estimado utilizando el método del inventario permanente a partir de los flujos de formación bruta de capital fijo, el cual calcula el conjunto del capital como una suma ponderada de las inversiones pasadas y se expresa en millones de euros a precios constantes. Los datos han sido obtenidos del INE. La vida útil estimada de los activos para la estimación de su depreciación anual ha sido la utilizada por Prados y Rosés (2008), y la tasa de crecimiento de la inversión neta utilizada para el 
cálculo del capital del primer periodo fue el promedio del crecimiento de inversión bruta para la totalidad de la serie.

Trabajo (L). Se estima a partir de los datos de la población económicamente activa en cada comunidad autónoma y para cada año. Los datos de esta variable se extraen de la encuesta de población activa (EPA) que está disponible a través del INE y figuran expresados en miles de personas.

Intensidad de capital o capital per cápita (K/L). Es una variable derivada de la relación entre el capital y la población económicamente activa en la comunidad autónoma; mide el stock de capital por unidad de factor trabajo, expresado en euros por trabajador activo.

Capital emprendedor (E). Estudiar la creación de nuevas empresas resulta complejo debido a la escasez de indicadores y a la existencia de una multiplicidad semántica para referirse a este concepto. Además, los índices efectivamente disponibles solo miden un espectro bastante limitado de potencial actividad empresarial, lo cual hace que la definición de la actividad emprendedora en un territorio sea aún más compleja. En esta línea, Marcotte (2013) estableció una clasificación de indicadores de actividad emprendedora.

Esta falta de un índice universalmente contrastado provoca una variabilidad de los resultados en función del que se utilice, por lo que resulta un problema añadido en las investigaciones.

Así pues, la obtención de esta variable implica la valoración de una serie de factores cualitativos que son difíciles de evaluar cuantitativamente; sin embargo, un indicador significativo del espíritu empresarial es la puesta en marcha de nuevas empresas. En consecuencia, se medirá el E de una comunidad como el número de nuevas empresas en relación a su población activa. Como sostienen Audretsch y Keilbach (2004b), si todo lo demás se mantiene constante, una mayor tasa de creación de empresas refleja mayores niveles de capital emprendedor. No obstante, siendo correcta esta afirmación, estos mismos autores apuntan hacia las iniciativas empresariales de mayor valor añadido en el ámbito de las nuevas tecnologías, como las que tienen un mayor impacto en el ámbito económico. Esta aserción fue corroborada por Callejón y Ortún (2009), quienes señalan que el crecimiento de la tasa de autoempleo por necesidad es un indicador negativo en una economía. En nuestro caso, todo se desarrollará en el contexto de las actividades planteado por Sánchez y Arellano (2012), en cuanto a actividades relacionadas con la economía del conocimiento y, además del indicador global $\mathrm{E}$, se utilizará la clasificación derivada del mismo ya comentada. Los datos fueron obtenidos de la base de datos del DIRCE.

Capital intelectual (IC). Como se ha señalado, en los diferentes modelos de IC aplicados a las regiones hay una carencia de metodologías contrastadas y la mayor parte de las propuestas existentes están limitadas a una recopilación sistemática de datos sin un marco de referencia comparativo. Asimismo, se detecta también otra dificultad añadida basada en que tales análisis comparativos se sustentan en los diferentes criterios de calidad y contenidos de los sistemas estadísticos regionales y nacionales (Klein, 2000). En el presente estudio, además de estas dificultades señaladas, hay que añadir la necesidad de conseguir un indicador de IC de carácter sintético, en la medida que este se incorporará como valor de las funciones del modelo.
En este sentido, el enfoque macroeconómico del modelo para la medición del IC propuesto por López et al. (2008), utilizado para medir el indicador regional de IC de las regiones de la UE-15 y su relación con el crecimiento y el PIB de cada una de ellas, se muestra como un indicador idóneo, tanto por su carácter de indicador sintético como por su enfoque macroeconómico, y además, por estar testado en un ámbito geográfico y metodológico muy similar al objeto de este estudio.

La ecuación de partida de este modelo, testado previamente en el ámbito empresarial, asume como premisa que, dada la complejidad del concepto teórico, difícilmente se pueden explicitar todos y cada uno de los componentes del IC. En consecuencia, el IC de cada uno de los territorios (ICi) será:

$\mathrm{IC}_{\mathrm{i}}=\mathrm{HC}_{\mathrm{i}}+\mathrm{SC}_{\mathrm{i}}+\mathrm{NEC}_{\mathrm{i}}$

donde $\mathrm{HC}_{\mathrm{i}}$ es el capital humano de cada región; se refiere a los conocimientos, las aptitudes, la motivación, la formación, etc., de los trabajadores así como al sistema de remuneración y la política de contratación; incluye masa salarial (salarios), inversión en educación (gasto público en educación por administración), índice de población con estudios superiores (porcentaje de personas con una edad comprendida entre 25-64 años con formación superior), tasa de actividad (tasa de actividad que se extrae de la encuesta de población activa).

$\mathrm{SC}_{i}$ es el capital estructural de cada región, definido como el conjunto de los capitales de procesos internos; incluye inversión en $\mathrm{I}+\mathrm{D}+\mathrm{i}$ (gasto total tanto público como privado), intensidad investigadora (indicador derivado, que se ha calculado como el cociente entre la inversión en I+D+i y el valor agregado bruto de cada comunidad), índice de penetración de internet en los hogares, índice de uso de PC con conexión a internet por los empleados e índice de utilización de comercio electrónico - «compras» $y$ «ventas»-. NEC $\mathrm{N}_{i}$ es el capital no explicitado, y se refiere a los capitales humanos y estructurales no incluidos en los anteriores.

En la tabla 2 se realiza un análisis descriptivo de las variables con el objetivo de obtener una visión general de estas. Para ello se han elegido como indicadores la media, la mediana, el mínimo, el máximo, la desviación típica y el coeficiente de variación, los cuales definen los valores y la variación de la serie a lo largo del periodo.

Con respecto a las variables dependientes, se puede observar en primer lugar como $\mathrm{Y}$ es una variable con una gran diferencia entre la mediana y la media, así como entre el valor mínimo y el máximo. Aparece también un alto nivel de dispersión que se manifiesta a través de su desviación típica y su coeficiente de variación. Todo esto representa grandes diferencias entre los niveles de $Y$ de cada una de las CCAA, además de una muy positiva evolución a lo largo del periodo. Sin embargo, cuando se observa la relación entre la actividad económica y la población activa $(\mathrm{Y} / \mathrm{L})$, estas grandes diferencias aparecen suavizadas, tal y como indica la similitud en los valores de la media y de la mediana, lo cual se ve confirmado a través de la observación de la desviación típica y el coeficiente de variación con valores muy moderados. Esta variación es debida fundamentalmente a la existencia 
Tabla 2 Análisis descriptivo de las variables

\begin{tabular}{lllllll}
\hline Variable & Media & Mediana & Mínimo & Máximo & Desv. típica & $\begin{array}{c}\text { Coeficiente } \\
\text { de variación }\end{array}$ \\
\hline IC & 0,17 & 0,16 & 0,10 & 0,28 & 0,04 & 0,24 \\
E & 0,81 & 0,81 & 0,00 & 1,77 & 0,31 & 0,39 \\
Sin_asal & 2,43 & 2,47 & 0,00 & 7,83 & 0,36 & 0,15 \\
de_1_a_2 & 0,43 & 0,42 & 0,00 & 1,59 & 0,13 & 0,30 \\
de_2_a_5 & 0,10 & 0,10 & 0,00 & 0,61 & 0,04 & 0,42 \\
de_6_a_9 & 0,13 & 0,13 & 0,00 & 0,27 & 0,03 & 0,24 \\
de_10_a_19 & 0,11 & 0,11 & 0,00 & 0,21 & 0,03 & 0,29 \\
de_20_a_49 & 0,06 & 0,06 & 0,00 & 0,18 & 0,02 & 0,38 \\
de_50_a_99 & 0,03 & 0,03 & 0,00 & 0,07 & 0,01 & 0,26 \\
K (miles) & $27.582 .000,00 €$ & $18.116 .000,00 €$ & $3.000 .000,00 €$ & $11.0600 .000,00 €$ & $26.669 .000,00 €$ & $0,97 €$ \\
L & $1.186 .800,00$ & $793.000,00$ & $116.980,00$ & $3.840 .400,00$ & $1.072 .600,00$ & 0,90 \\
(Y/L) (miles) & $37.712,20$ & $37.181,80$ & $23.829,40$ & $57.013,70$ & $6.783,26$ & 0,18 \\
Y (millones) & $4.524 .320,00 €$ & $2.698 .800,00 €$ & $465.110,00 €$ & $18.405 .400,00 €$ & $4.420 .470,00 €$ & $0,98 €$ \\
K/L & $23.995,10$ & $22.562,40$ & $9.483,70$ & $43.687,90$ & $7.576,31$ & 0,32 \\
\hline
\end{tabular}

Fuente: elaboración propia.

de un grupo de comunidades fuertemente diferenciadas del resto en este aspecto.

Con respecto a las variables independientes, tanto $\mathrm{K}$ como $L$ siguen un esquema muy similar a la actividad económica, con grandes diferencias entre la media y la mediana, el valor mínimo y el máximo, y un altísimo coeficiente de variación. Las razones vuelven a ser las mismas diferencias entre CCAA y crecimiento del periodo que se apuntaba en el caso de Y. De igual manera, la relación entre capital y población activa $(\mathrm{K} / \mathrm{L})$ sigue un patrón ajustado a lo que ocurría con $Y / L$, si bien, en este caso, el coeficiente de variación es más alto, lo que parece indicar una mayor diferencia en la dotación de capital entre CCAA de lo que ocurría con el nivel de actividad económica. Por su parte, en referencia a la variable IC, se puede observar que tiene una media ligeramente superior a la mediana y un bajo nivel de desviación típica que da lugar a un moderado coeficiente de variación. Dado que se trata de una serie temporal, se puede intuir que dicha variable tendrá un crecimiento progresivo y sostenido a lo largo del tiempo. Finalmente, E presenta una estructura de indicadores similar al IC, comprobándose una tendencia positiva a lo largo del periodo, con una media igual a la mediana y un importante nivel de desviación típica, lo que da lugar a un alto coeficiente de variación. Dado el carácter de la serie, se intuye igualmente un crecimiento sostenido de esta variable.

Esta alta sensibilidad de la variable E y sus subvariables en función del tamaño apuntan a una cierta debilidad de la misma. Al observar sus coeficientes de variación y dispersión, se confirma la alta variabilidad de las observaciones, que no responden a ningún tipo de patrón temporal o transversal. Esto resulta sorprendente ya que, admitiendo diferencias en cuanto al comportamiento de la variable en diferentes contextos territoriales -basadas en las características endógenas de cada una de ellas-, estas deberían responder a patrones de comportamiento interno y diferenciado de otros territorios, o bien responder a un patrón temporal común al conjunto de las CCAA, como consecuencia de una coyuntura socioeconómica común, salvo que existan factores exógenos como, por ejemplo, políticas públicas de apoyo a la empresa. Esta debilidad puede hacer más compleja la obtención de un resultado significativamente estadístico, en la medida que crece la exigencia de las técnicas estadísticas a utilizar, pero no niega de forma radical el fenómeno de estudio, sino que hace más difícil la constatación matemática del mismo.

\subsection{Metodología de análisis}

Para contrastar las hipótesis propuestas se ha construido un panel de datos. Por su parte, para la estimación de los parámetros de las funciones y por la composición de la información (dimensión temporal y estructural) se ha utilizado la técnica de regresiones de datos de panel, tratando de encontrar la heterogeneidad no observable entre CCAA.

La naturaleza de los datos sugiere que se debe utilizar un modelo de efectos fijos, dado que las unidades transversales no pueden ser consideradas muestras aleatorias de una gran población, porque corresponden a todas las CCAA españolas. Por lo tanto, como señala Wooldridge (2002), es razonable pensar en los efectos inobservables como parámetros a estimar y, por ende, en modelos de efectos fijos. No obstante, para confirmar la idoneidad de este método de estimación se ha llevado a cabo el test de Hausman; al rechazar la hipótesis nula, la estimación mediante efectos fijos es válida.

Para corregir la heterocedasticidad se ha utilizado el método de estimación de los mínimos cuadrados ponderados (MCP). En este sentido, en presencia de heterocedasticidad, la estimación por MCP proporciona un estimador más eficiente que el de los mínimos cuadrados ordinarios (MCO) y estadísticos de prueba válidos cuando el término de error se distribuye como una normal (Wooldridge, 2002).

Así pues, siguiendo el razonamiento de Audretsch y Keilbach (2004b), se plantean 2 funciones econométricas. La primera trata de medir el impacto del capital emprendedor en la actividad económica de cada comunidad autónoma, para lo que se utilizará una función del tipo Cobb y Douglas 
(1928), tomando como base el modelo de crecimiento endógeno:

$Y_{i t}=\alpha K_{i t}^{\beta 1} L_{i t}^{\beta 2} C l_{i t}^{\beta 3} E_{i t}^{\beta 4} e^{\varepsilon i t}$

donde, $Y_{i t}$ es el nivel de actividad económica de la comunidad autónoma $i$ en el periodo $t ; K_{i t}$ es el stock de capital fijo de la comunidad autónoma $i$ en el periodo $t ; L_{i t}$ es el número de trabajadores activos de la comunidad autónoma $i$ en el periodo $t ; C_{i t}$ es IC de la comunidad autónoma $i$ en el periodo $t$; $E_{i t}$ representa el capital emprendedor de la comunidad autónoma $i$ en el periodo $t$; $e$ un factor de error que agrupa otras variables no controladas en el modelo.

La segunda función estima sus repercusiones en la productividad laboral de cada comunidad autónoma. Se trata de la función que representa la productividad laboral $\left(Y_{i t} / L_{i t}\right)$ de la comunidad autónoma $i$ en el período $t$ :

$$
\left(Y_{i t} / L_{i t}\right)=\alpha\left(K_{i t} / L_{i t}\right)^{\beta 1} C l_{i t}^{\beta 2} E_{i t}^{\beta 3} e^{\varepsilon i t}
$$

Ambas funciones asumen 2 interpretaciones simultáneas en los parámetros. Un valor positivo de $\beta 1$ es consistente con la teoría neoclásica del crecimiento, mientras que un valor positivo de $\beta 2$ y los otros $\beta \mathrm{j}$ son consistentes con la teoría del crecimiento endógeno.

\section{Resultados}

En la tabla 3 se muestran los resultados de la regresión, la cual está basada en la función 1, en la que se utilizan el IC y el E como variables explicativas. El resultado de la primera regresión muestra a $\mathrm{K}$ y $\mathrm{L}$ como únicas variables explicativas de la actividad económica, coincidiendo con los modelos neoclásicos de crecimiento económico. Los coeficientes $\beta 0$ y $\beta 1$ son estadísticamente significativos, con valores de 0,50 y 0,51 , respectivamente, lo que resulta consistente con los resultados obtenidos por Masson (2007) y Pulido y RodríguezVález (2006).

La segunda regresión integra el factor conocimiento a través del IC, coincidiendo con los modelos de crecimiento endógeno. Dichos modelos consideran que este nuevo factor puede generar rendimientos de escala crecientes y efectos de propagación con capacidad de promover el crecimiento económico. En el presente estudio se comprueba que $\beta 2$ es estadísticamente significativo al $1 \%$ en todas las regresiones en las que participa, con un valor de entre 0,27 y 0,28.

La evidencia sobre la relación entre creación de empresas y actividad económica es constatada por Fölster (2000), Acs (2007) y Bosma (2009), entre otros, si bien estos autores establecen ciertas diferencias con respecto al tipo de emprendedor, la oportunidad o necesidad del emprendimiento y el tamaño de la empresa. En el presente estudio, cuando se analiza la incorporación del E, y a la vista de los resultados de $\beta 3$ en las regresiones tercera a décima, se comprueba que el $E$ global y el de los emprendedores sin asalariados no son estadísticamente significativos. Es preciso mencionar que el colectivo de empresas sin asalariados supone el 52,32\% del total, por lo que el comportamiento de ambas variables es muy similar; esto se confirma al observar la similitud de los valores de sus coeficientes y de sus $\rho$.
Por su parte, cuando se analiza dicha variable a través del resto de sus segmentos, se constata que el segmento de 3 a 5 empleados sería significativo al $10 \%$ y con un coeficiente de 0,0162454. El segmento de 6 a 9 empleados no sería significativo al $10 \%$, si bien sí lo sería al $17,5 \%$. Al agrupar estos 2 segmentos en uno nuevo que incluya a las empresas de 3 a 9 empleados, se mejora de forma considerable el coeficiente 0,0315914, y así resulta significativo al $5 \%$. Por su parte, con el segmento de 10 a 19 empleados el comportamiento es similar, es decir, a pesar de existir un coeficiente positivo no resulta significativo; sin embargo, cuando se amplía el rango en un nuevo segmento y se incluyen las empresas de 3 a 19 empleados, el resultado es significativo al $10 \%$ y su coeficiente es del 0,0323787.

En la tabla 4 se muestran los resultados de las regresiones basadas en la función 2, en la que se utilizan el IC y el E como variables explicativas de la productividad laboral. En la primera regresión de la tabla se puede observar que $\beta 1$ es positivo y es estadísticamente significativo al $1 \%$, con un valor de 0,47753 , el cual es ligeramente superior al obtenido por Audretsch y Keilbach (2004a), quienes también trabajaron en esta misma línea. Con respecto al factor conocimiento $\beta 2$, es positivo y estadísticamente significativo al $1 \%$ en todas las regresiones planteadas, lo que es consistente con la teoría del crecimiento endógeno (Romer, 1986, 1990; Lucas, 1988).

Al igual que ocurría en la función 1 y a la vista de los resultados de $\beta 3$ en las regresiones tercera a décima, la relación entre $\mathrm{E}$ y productividad laboral es positiva y estadísticamente significativa al $10 \%$ en el segmento de 3 a 5 asalariados, con un coeficiente de 0,014636. De igual manera, el segmento de 6 a 9 empleados tiene un coeficiente positivo que, aun no siendo significativo al $10 \%$, sí lo sería al $17,8 \%$. Al agrupar estos 2 segmentos en uno nuevo que incluya a las empresas de 3 a 9 empleados se replican los resultados obtenidos anteriormente y mejora considerablemente el coeficiente a 0,0292665, resultando significativo al $5 \%$. Con respecto al segmento de 10 a 19 empleados, vuelve a ocurrir que a pesar de existir un coeficiente positivo, el resultado no resulta significativo; sin embargo, cuando se amplía el rango en un nuevo segmento y se incluye a las empresas de 3 a 19 empleados, el resultado es significativo al $10 \%$ y su coeficiente es del 0,0311184 . Por su parte, el segmento de 1 a 2 asalariados presenta un coeficiente negativo, que en este caso sí es significativo al 10\%, a diferencia del apartado anterior. Esto puede relacionarse con el tamaño mínimo requerido de las empresas y el carácter de necesidad y oportunidad de la actividad emprendedora. Para concluir, se constata igualmente que el impacto de la variable $E$ en la variable dependiente $Y / L$ es muy inferior al de la variable IC, lo que está en línea con los resultados obtenidos por Audretsch y Keilbach (2004a) y Masson (2007).

En la tabla 5 se puede comprobar el conjunto de los resultados para las hipótesis planteadas.

\section{Discusión y conclusiones}

En el presente trabajo se ha analizado cuál ha sido el impacto, desde un punto de vista económico, de la acumulación de IC y E en la primera década del sigloxXI, con el 
Tabla 3 Regresión función 1, actividad económica

\begin{tabular}{|c|c|c|c|c|c|c|c|c|c|c|c|c|c|c|c|c|c|c|c|c|c|c|c|c|}
\hline & Coef & $\mathrm{p}$ & Coef & $p$ & Coef & $p$ & Coef & $p$ & Coef & $p$ & Coef & p & Coef & $p$ & Coef & $p$ & Coef & $\mathrm{p}$ & Coef & p & Coef & $p$ & Coef & $\mathrm{p}$ \\
\hline Constante & $\begin{array}{l}5,44520 \\
<0,00001\end{array}$ & $\ldots * *$ & $\begin{array}{l}7,50869 \\
<0,00001\end{array}$ & $* * *$ & $\begin{array}{l}7,51370 \\
<0,00001\end{array}$ & $\ldots$ & $\begin{array}{l}7,45705 \\
<0,00001\end{array}$ & $\ldots$ & $\begin{array}{l}7,40876 \\
<0,00001\end{array}$ & $\cdots *$ & $\begin{array}{l}7,55805 \\
<0,00001\end{array}$ & $* * *$ & $\begin{array}{l}7,60146 \\
<0,00001\end{array}$ & $\ldots$ & $\begin{array}{l}7,59458 \\
<0,00001\end{array}$ & $\ldots$ & $\begin{array}{l}7,59693 \\
<0,00001\end{array}$ & $\ldots$ & $\begin{array}{l}7,56918 \\
<0,00001\end{array}$ & *** & $\begin{array}{l}7,51163 \\
<0,00001\end{array}$ & $* *$ & $\begin{array}{l}7,50650 \\
<0,00001\end{array}$ & $\ldots$ \\
\hline $\ln \mathrm{K}$ & $\begin{array}{l}0,499162 \\
<0,00001\end{array}$ & $* *$ & $\begin{array}{l}0,352927 \\
<0,00001\end{array}$ & $\ldots * *$ & $\begin{array}{l}0,351594 \\
<0,00001\end{array}$ & $* * *$ & $\begin{array}{l}0,264303 \\
<0,00001\end{array}$ & $* * *$ & $\begin{array}{l}0,359985 \\
<0,00001\end{array}$ & *** & $\begin{array}{l}0,350521 \\
<0,00001\end{array}$ & *** & $\begin{array}{l}0,352068 \\
<0,00001\end{array}$ & *.. & $\begin{array}{l}0,349321 \\
<0,00001\end{array}$ & $* *$ & $\begin{array}{l}0,349635 \\
<0,00001\end{array}$ & *** & $\begin{array}{l}0,350936 \\
<0,00001\end{array}$ & *** & $\begin{array}{l}0,354389 \\
<0,00001\end{array}$ & *** & $\begin{array}{l}0,353248 \\
<0,00001\end{array}$ & $\ldots$ \\
\hline $\ln \mathrm{L}$ & $\begin{array}{l}0,512135 \\
<0,00001\end{array}$ & $\ldots$ & $\begin{array}{l}0,642622 \\
<0,00001\end{array}$ & $\ldots$ & $\begin{array}{l}0,644696 \\
<0,00001\end{array}$ & $\ldots$ & $\begin{array}{l}0,356535 \\
<0,00001\end{array}$ & $\ldots$ & $\begin{array}{l}0,635128 \\
<0,00001\end{array}$ & $\ldots$ & $\begin{array}{l}0,644981 \\
<0,00001\end{array}$ & $\ldots$ & $\begin{array}{l}0,64216 \\
<0,00001\end{array}$ & .** & $\begin{array}{l}0,646208 \\
<0,00001\end{array}$ & $\ldots$ & $\begin{array}{l}0,644711 \\
<0,00001\end{array}$ & *** & $\begin{array}{l}0,644718 \\
<0,00001\end{array}$ & $\ldots$ & $\begin{array}{l}0,640167 \\
<0,00001\end{array}$ & $\cdots$ & $\begin{array}{l}0,641986 \\
<0,00001\end{array}$ & $\ldots$ \\
\hline $\ln \mathrm{IC}$ & & & $\begin{array}{l}0,276905 \\
<0,00001\end{array}$ & $\ldots$ & $\begin{array}{l}0,272393 \\
<0,00001\end{array}$ & $\ldots$ & $\begin{array}{l}0,638952 \\
<0,00001\end{array}$ & $\ldots$ & $\begin{array}{l}0,271088 \\
<0,00001\end{array}$ & $\ldots$ & $\begin{array}{l}0,269302 \\
<0,00001\end{array}$ & $\ldots$ & $\begin{array}{l}0,286015 \\
<0,00001\end{array}$ & $\ldots$ & $\begin{array}{l}0,278432 \\
<0,00001\end{array}$ & $\ldots$ & $\begin{array}{l}0,282444 \\
<0,00001\end{array}$ & $\ldots$ & $\begin{array}{l}0,280110 \\
<0,00001\end{array}$ & ... & $\begin{array}{l}0,278526 \\
<0,00001\end{array}$ & $\ldots$ & $\begin{array}{l}0,280402 \\
<0,00001\end{array}$ & $\ldots$ \\
\hline $\ln E$ & & & & & $\begin{array}{l}0,0107955 \\
0,38165\end{array}$ & & & & & & & & & & & & & & & & & & & \\
\hline $\begin{array}{l}\text { In E sin } \\
\quad \text { asalariados }\end{array}$ & & & & & & & $\begin{array}{l}0,0114576 \\
0,32971\end{array}$ & & & & & & & & & & & & & & & & & \\
\hline In E de 1 a 2 & & & & & & & & & $\begin{array}{l}-0,0258004 \\
0,13527\end{array}$ & & & & & & & & & & & & & & & \\
\hline $\ln E$ de 3 a 5 & & & & & & & & & & & $\begin{array}{l}0,0162454 \\
0,06746\end{array}$ & & & & & & & & & & & & & \\
\hline $\ln E$ de 6 a 9 & & & & & & & & & & & & & $\begin{array}{l}0,0277468 \\
0,17780\end{array}$ & & & & & & & & & & & \\
\hline $\ln E$ de 3 a 9 & & & & & & & & & & & & & & & $\begin{array}{l}0,0315914 \\
0,0370\end{array}$ & $*$ & & & & & & & & \\
\hline $\begin{array}{l}\text { In E de } 10 \\
\text { a } 19\end{array}$ & & & & & & & & & & & & & & & & & 0,0167294 & & & & & & & \\
\hline In E de 2 a 19 & & & & & & & & & & & & & & & & & 0,28835 & & $\begin{array}{l}0,0323787 \\
0,0860\end{array}$ & * & & & & \\
\hline $\begin{array}{c}\text { In E de } 20 \\
\text { a } 49\end{array}$ & & & & & & & & & & & & & & & & & & & & & $\begin{array}{l}0,00231368 \\
0,83354\end{array}$ & & & \\
\hline $\begin{array}{c}\text { In E de } 50 \\
\text { a } 99\end{array}$ & & & & & & & & & & & & & & & & & & & & & & & $\begin{array}{l}-0,00232899 \\
0,90407\end{array}$ & \\
\hline
\end{tabular}


Tabla 4 Regresión función 2, productividad laboral

\begin{tabular}{|c|c|c|c|c|c|c|c|c|c|c|c|c|c|c|c|c|c|c|c|c|c|c|c|c|}
\hline \multirow{2}{*}{ Constante } & Coef & $p$ & Coef & $p$ & Coef & $\mathrm{p}$ & Coef & $\mathrm{p}$ & Coef & $p$ & Coef & $p$ & Coef & $p$ & Coef & $p$ & Coef & p & Coef & $p$ & Coef & $\mathrm{p}$ & Coef & $p$ \\
\hline & $\begin{array}{l}5,73502 \\
<0,00001\end{array}$ & 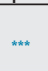 & $\begin{array}{l}7,38466 \\
<0,00001\end{array}$ & 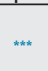 & $\begin{array}{l}7,38084 \\
<0,00001\end{array}$ & $\ldots$ & $\begin{array}{l}7,30182 \\
<0,00001\end{array}$ & $\ldots$ & $\begin{array}{l}7,24863 \\
<0,00001\end{array}$ & $\ldots$ & $\begin{array}{l}7,42073 \\
<0,00001\end{array}$ & $\cdots$ & $\begin{array}{l}7,43247 \\
<0,00001\end{array}$ & $\ldots$ & $\begin{array}{l}7,46787 \\
<0,00001\end{array}$ & "** & $\begin{array}{l}7,42029 \\
<0,00001\end{array}$ & *** & $\begin{array}{l}7,44713 \\
<0,00001\end{array}$ & ** & $\begin{array}{l}7,35572 \\
<0,00001\end{array}$ & $\cdots$ & $\begin{array}{l}7,37853 \\
<0,00001\end{array}$ & $\ldots$ \\
\hline $\ln \mathrm{K} / \mathrm{L}$ & $\begin{array}{l}0,47753 \\
<0,00001\end{array}$ & $\ldots$ & $\begin{array}{l}0,360691 \\
<0,00001\end{array}$ & $\ldots$ & $\begin{array}{l}0,263678 \\
<0,00001\end{array}$ & $\ldots$ & $\begin{array}{l}0,366852 \\
<0,00001\end{array}$ & $\ldots$ & $\begin{array}{l}0,370344 \\
<0,00001\end{array}$ & $\ldots$ & $\begin{array}{l}0,359204 \\
<0,00001\end{array}$ & $\ldots$ & $\begin{array}{l}0,362628 \\
<0,00001\end{array}$ & $\ldots$ & $\begin{array}{l}0,357086 \\
<0,00001\end{array}$ & *** & $\begin{array}{l}0,361126 \\
<0,00001\end{array}$ & $\cdots$ & $\begin{array}{l}0,358484 \\
<0,00001\end{array}$ & $\cdots$ & $\begin{array}{l}0,363453 \\
<0,00001\end{array}$ & $\ldots$ & $\begin{array}{l}0,359663 \\
<0,00001\end{array}$ & .t. \\
\hline $\ln I C$ & & & $\begin{array}{l}0,269273 \\
<0,00001\end{array}$ & $m$ & $\begin{array}{l}0,360555 \\
<0,00001\end{array}$ & $* *$ & $\begin{array}{l}0,254095 \\
<0,00001\end{array}$ & $* *$ & $\begin{array}{l}0,26133 \\
<0,00001\end{array}$ & *** & $\begin{array}{l}0,261461 \\
<0,00001\end{array}$ & $* *$ & $\begin{array}{l}0,274069 \\
<0,00001\end{array}$ & $* *$ & $\begin{array}{l}0,271248 \\
<0,00001\end{array}$ & $\ldots$ & $\begin{array}{l}0,270436 \\
<0,00001\end{array}$ & $\ldots$ & $\begin{array}{l}0,272716 \\
<0,00001\end{array}$ & ".* & $\begin{array}{l}0,268812 \\
<0,00001\end{array}$ & $\ldots * *$ & $\begin{array}{l}0,274581 \\
<0,00001\end{array}$ & $*$ \\
\hline $\ln \mathrm{E}$ & & & & & $\begin{array}{l}0,0072034 \\
0,55578\end{array}$ & & & & & & & & & & & & & & & & & & & \\
\hline $\begin{array}{l}\text { In E sin } \\
\text { asalariados }\end{array}$ & & & & & & & $\begin{array}{l}0,0082323 \\
0,47514\end{array}$ & & & & & & & & & & & & & & & & & \\
\hline In $E$ de 1 a 2 & & & & & & & & & $\begin{array}{l}-0,0299734 \\
0,06922\end{array}$ & . & & & & & & & & & & & & & & \\
\hline In $E$ de 3 a 5 & & & & & & & & & & & $\begin{array}{l}0,014636 \\
0,08634\end{array}$ & . & & & & & & & & & & & & \\
\hline In $E$ de 6 a 9 & & & & & & & & & & & & & $\begin{array}{l}0,0277536 \\
0,17863\end{array}$ & & & & & & & & & & & \\
\hline $\ln E$ de 3 a 9 & & & & & & & & & & & & & & & $\begin{array}{l}0,0292665 \\
0,0490\end{array}$ & $* *$ & & & & & & & & \\
\hline $\begin{array}{l}\text { In E de } 10 \\
\text { a } 19\end{array}$ & & & & & & & & & & & & & & & & & 0,0170345 & & & & & & & \\
\hline In E de 3 a 19 & & & & & & & & & & & & & & & & & 0,25329 & & $\begin{array}{l}0,0311184 \\
0,0880\end{array}$ & 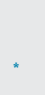 & & & & \\
\hline $\begin{array}{c}\text { In E de } 20 \\
\text { a } 49\end{array}$ & & & & & & & & & & & & & & & & & & & & & $-0,00047597$ & & & \\
\hline $\begin{array}{c}\text { In E de } 50 \\
\text { a } 99\end{array}$ & & & & & & & & & & & & & & & & & & & & & & & $\begin{array}{l}-0,00767832 \\
0,68104\end{array}$ & \\
\hline
\end{tabular}


Tabla 5 Resultados del contraste de hipótesis

\begin{tabular}{|c|c|c|c|c|}
\hline \multirow[b]{2}{*}{ Hipótesis } & \multicolumn{4}{|c|}{ Resultados } \\
\hline & Esperado & Obtenido & Significación & Validación \\
\hline \multicolumn{5}{|l|}{$H 1$} \\
\hline+ & + & $* * *$ & Aceptada & \\
\hline \multicolumn{5}{|l|}{$H 2$} \\
\hline+ & + & $* * *$ & Aceptada & \\
\hline \multicolumn{5}{|l|}{ H3 } \\
\hline+ & + & & Rechazada & \\
\hline $\mathrm{H} 3_{\mathrm{A}}$ & + & + & & Rechazada \\
\hline $\mathrm{H} 3_{\mathrm{B}}$ & + & + & & Rechazada \\
\hline $\mathrm{H} 3_{\mathrm{C}}$ & + & + & * & Aceptada \\
\hline $\mathrm{H} 3_{\mathrm{D}}$ & + & + & & Rechazada \\
\hline $\mathrm{H} 3_{\mathrm{E}}$ & + & + & ** & Aceptada \\
\hline $\mathrm{H} 3_{\mathrm{F}}$ & + & + & & Rechazada \\
\hline $\mathrm{H} 3_{\mathrm{G}}$ & + & + & * & Aceptada \\
\hline $\mathrm{H} 3_{\mathrm{H}}$ & + & + & & Rechazada \\
\hline $\mathrm{H} 3 \mathrm{I}$ & + & - & & Rechazada \\
\hline \multicolumn{5}{|l|}{$\mathrm{H} 4$} \\
\hline+ & + & & Rechazada & \\
\hline $\mathrm{H} 4_{\mathrm{A}}$ & + & + & & Rechazada \\
\hline $\mathrm{H} 4_{\mathrm{B}}$ & + & - & * & Rechazada \\
\hline $\mathrm{H} 4_{\mathrm{C}}$ & + & + & * & Aceptada \\
\hline $\mathrm{H} 4_{D}$ & + & + & & Rechazada \\
\hline $\mathrm{H} 4_{\mathrm{E}}$ & + & + & ** & Aceptada \\
\hline $\mathrm{H} 4_{\mathrm{F}}$ & + & + & & Rechazada \\
\hline $\mathrm{H} 4_{\mathrm{G}}$ & + & + & * & Aceptada \\
\hline $\mathrm{H} 4_{\mathrm{H}}$ & + & - & & Rechazada \\
\hline $\mathrm{H} 4$ & + & - & & Rechazada \\
\hline
\end{tabular}

fin de conocer en qué medida estos 2 elementos pueden ser factores impulsores de crecimiento regional en España.

A la vista de los resultados obtenidos, se puede observar una gran diferencia entre IC y E con respecto a los resultados esperados, siendo más relevante el conocimiento que la creación de empresas para explicar la actividad económica y la productividad laboral. Por su parte, la variable IC se muestra fuertemente correlacionada y estadísticamente significativa en ambas funciones y en todas las regresiones realizadas con los diferentes tramos de la variable $\mathrm{E}$, mostrando asimismo un valor muy estable en todos los casos. De esta forma, se comprueba que el capital humano y el capital físico son elementos necesarios, pero no suficientes, para generar crecimiento económico (Holcombe, 1998), y se puede afirmar que la diseminación del conocimiento en una economía se configura como un elemento importante. Así mismo, se confirma la idoneidad del indicador utilizado, IC, para recoger y representar la cantidad existente de este factor.

Este carácter instrumental del $\mathrm{E}$ es el que se pone de manifiesto a través de las hipótesis $\mathrm{H}_{3}$ y $\mathrm{H}_{4}$. Los resultados demuestran que el $\mathrm{E}$ se convierte en un agente capaz de convertir la existencia de spillovers de conocimiento en crecimiento económico, a través de su transformación en actividades comerciales (Arrow, 1962).

No obstante, en el ámbito de la economía del conocimiento, objeto del presente estudio, este E con capacidad para generar mayor actividad económica y productividad se encuentra concentrado entre las empresas nacientes con un menor número de empleados, concretamente entre las que tienen entre 3 y 19 empleados, que es el segmento que se muestra estadísticamente significativo, coincidiendo con los resultados obtenidos por Masson (2007), si bien el ya citado carácter específico de estas empresas, y su todavía escasa implantación en nuestra economía, condicionan los resultados.

Una de las diferencias observables respecto a otros estudios es la baja correlación que el segmento de 1 a 2 empleados tiene con la actividad económica, y especialmente con la productividad, llegando a ser con esta última negativa y estadísticamente significativa. Una posible explicación podría ser la alta cualificación de los profesionales que las componen, que por sí solas no puede crear conocimiento económicamente explotable, sino que necesitan establecer nuevas percepciones, pensamientos y experiencias en el contexto de una organización (Nonaka y Takeuchi, 1995; Stewart, 1997).

Por otro lado, estos resultados confirman la idea de que en contextos en los que existe un alto nivel de actividad emprendedora se fomenta también la innovación y la competencia a través de la rotación empresarial, eliminando las menos eficientes y fomentando las que lo son más (Callejón y Segarra, 1999), si bien el segmento de empresas que resulta significativamente estadístico es todavía el $13,77 \%$ del total de empresas creadas en el ámbito de la economía del conocimiento.

En definitiva, y a la luz de los resultados, se comprueba que $E$ es un agente para la trasformación y la distribución del conocimiento, logrando convertirlo en económicamente comercializable. Por otro lado, el flujo neto de creación de empresas es un proceso que genera mejoras en la productividad y facilita la modernización de la estructura empresarial.

\subsection{Implicaciones prácticas}

Los resultados obtenidos en el presente estudio confirman que $E$ ejerce un impacto positivo sobre el crecimiento económico regional, si bien la dimensión de las empresas creadas se configura como un factor fundamental a la hora de determinar dicho impacto, lo cual tiene importantes implicaciones prácticas para los emprendedores y para las administraciones públicas. En este sentido, es oportuno señalar que es preciso fomentar y difundir la cultura emprendedora, y proteger especialmente a las micro y pequeñas empresas en el ámbito de la economía del conocimiento, ya que son precisamente estas las que más contribuyen al incremento de la actividad económica y a la generación de riqueza regional. Así, es importante que se produzca un mayor apoyo institucional y reconocimiento social al emprendimiento con la finalidad de alentar proyectos innovadores que perduren en el tiempo y sean capaces de generar empleo y riqueza. En definitiva, dada la grave y larga crisis económica, con agudas consecuencias sociales, que ha atravesado nuestro país desde el año 2008 y que 
actualmente presenta claros signos de recuperación, el presente trabajo ha pretendido contribuir en el avance de un tema tan necesario y oportuno como es el emprendimiento en la economía del conocimiento.

\subsection{Limitaciones y futuras líneas de investigación}

En cuanto a las limitaciones de la presente investigación, cabe destacar la dificultad de medición del $\mathrm{E}$, especialmente en este caso por la especificidad del tipo de empresas elegido, ya que en algunos segmentos el valor de la variable $\mathrm{E}$ resultó prácticamente inapreciable. Esta dificultad tiene su impacto negativo al integrar la variable en un modelo tan exigente como el de datos panel, condicionando los resultados con una variable tan frágil como $E$.

En este sentido, entre las futuras líneas de investigación se plantea precisamente la posibilidad de establecer indicadores alternativos que midan dicha variable. Se considera también la posibilidad de integrar, mediante un modelo econométrico que permita medir los efectos temporales de una variable, el carácter dinámico de E. Así mismo, establecer cuáles son los factores que favorecen o dificultan la aparición de $\mathrm{E}$ resultaría de sumo interés para determinar políticas públicas en esta dirección.

Profundizar en la relación entre la economía del conocimiento y el crecimiento económico, intentando encontrar conexiones entre la creación de nuevas empresas en el ámbito de la economía del conocimiento y la composición de la estructura económica regional, se configura como una posible alternativa de investigación, permitiendo de esta forma el establecimiento de planes más concretos por territorios a la hora de definir la promoción del espíritu emprendedor.

\section{Bibliografía}

Acs, Z.J., Armington, C., 2004. Employment growth and entrepreneurial activity in cities. Regional Studies 38, 911-927.

Acs Z.J., Audretsch D.B., Braunerhjelm P., Carlsson B., 2004. The missing link the knowledge filter and entrepreneurship in endogenous growth. Working Paper 4783. Center for Economic Policy Research, London.

Acs, Z.J., Szerb, L., 2007. Entrepreneurship, economic growth and public policy. Small Business Economics 28 (2-3), 109-122.

Acs, Z.J., Audretsch, D.B., Lehmann, E.E., 2013. The knowledge spillover theory of entrepreneurship. Small Business Economics 41, 757-774.

Arrow, K.J., 1962. The economic implications of learning by doing. Review of Economics Studies 29, 155-173.

Aubry, M., Bonnet, J., Renou-Maissant, P., 2015. Entrepreneurship and the business cycle: The "Schumpeter" effect versus the "refugee" effect-a French appraisal based on regional data. The Annals of Regional Science 54, 23-55.

Audretsch, D.B., 2007. Entrepreneurship capital and economic growth. Oxford Review of Economic Policy 23, 63-78.

Audretsch, D., 2009. Capital emprendedor y crecimiento económico. Investigaciones Regionales [monográfico], 27-45.

Audretsch, D., Feldman, M., 1996. R\&D spillovers and the geography of innovation and production. American Economic Review 86, 630-640.

Audretsch, D.B., Fritsch, M., 2002. Growth regimes over time and space. Regional Studies 36, 113-124.
Audretsch, D.B., Keilbach, M., 2004a. Entrepreneurship and regional growth: An evolutionary interpretation. Journal of Evolutionary Economics 14, 605-616.

Audretsch, D., Keilbach, M., 2004b. Entrepreneurship capital and economic performance. Regional Studies 38, 949-959.

Audretsch, D.B., Keilbach, M., 2005. Entrepreneurship capital and regional growth. Annals of Regional Science 39, 457-469.

Barrena-Martínez, J., López-Fernández, M., Romero-Fernández, P.M., 2016. Efectos de las políticas de recursos humanos socialmente responsables en el capital intelectual. Intangible Capital 12, 549-590.

Benito-Hernández, S., Platero-Jaime, M., Rodríguez-Duarte, A., 2012. Factores determinantes de la innovación en las microempresas españolas: La importancia de los factores internos. Universia Business Review 33, 104-121.

Bontis, N., 1998. Intellectual capital: An exploratory study that develops measures and models. Management Decision 36, 63-76.

Bosma, N., 2009. The Geography of Entrepreneurial Activity and Regional Economic Development. A-D Drukb.v., Zeist, Netherlands.

Bradley, K., 2003. Intellectual capital and the new wealth of nations II. Business Strategy Review 8, 33-44.

Bueno, E., 1998. El capital intangible como clave estratégica en la competencia actual. Boletín de Estudios Económicos 53, 207-229.

Callejón, M., Ortún, V., 2009. La caja negra de la dinámica empresarial. Investigaciones Regionales 15, 167-189.

Callejón, M., Segarra, A., 1999. Business dynamics and efficiency in industries and regions. The case of Spain. Small Business Economics 13, 253-271.

Carree, M.A., Thurik, A.R., 2006. The Handbook of Entrepreneurship and Economic Growth. International Library of Entrepreneurship, Cheltenham.

Castaño, M.S., Méndez, M.T., Galindo, M.A., 2016. The effect of public policies on entrepreneurial activity and economic growth. Journal of Business Research 69, 5280-5285.

Castilla-Polo, F., Gallardo-Vázquez, D., 2016. The main topics of research on disclosures of intangible assets: A critical review. Accounting, Auditing \& Accountability Journal 29, 323-356.

Cobb, C., Douglas, P., 1928. A theory production. The American Economic Review 18, 139-165.

Davenport, T., Prusak, O., 1997. Knowledege Management Glossary Information Ecology: Mastering the Information and Knowledge Enviroment. Oxford University Press, Oxford.

Davenport, T.H., Prusak, L., 1998. Working knowledge: How organizations manage what they know. Harvard Business Press, Harvard.

Delgado-Verde, M., Martín-de-Castro, G., Navas-López, J.E., Cruz-González, J., 2011. Capital social, capital relacional e innovación tecnológica. Una aplicación al sector manufacturero español de alta y media-alta tecnología. Cuadernos de Economía y Dirección de la Empresa 14, 207-221.

Edvinsson, L., Sullivan, P., 1996. Developing a model for managing intellectual capital. European Management Journal 14, 356-364.

Edvinsson, L., Malone, M.S., 1999. El capital intelectual. Cómo identificar y calcular el valor de los recursos intangibles de su empresa. Gestión 2000, Barcelona.

Fölster, S., 2000. Do entrepreneurs create jobs? Small Business Economics 14, 137-148.

Galindo, M.A., Méndez, M.T., 2014. Entrepreneurship, economic growth, and innovation: Are feedback effects at work? Journal of Business Research 67, 825-829.

García, C., Martínez, A., Fernández, R., 2010. Características del emprendedor influyentes en el proceso de creación empresarial y en el éxito esperado. Revista Europea de Dirección y Economía de la Empresa 19, 31-48.

Griliches, Z., 1979. Issues in assessing the contribution of R\&D to productivity growth. The Bell Journal of Economics 10, 92-116. 
Holcombe, R., 1998. Entrepreneurship and economic growth. Quarterly Review of Austrian Economics 1, 45-62.

Huggins, R., Thompson, P., 2015. Entrepreneurship, innovation and regional growth: A network theory. Small Business Economics 45, 103-128.

Jaffe, A., 1989. The real effects of academic research. American Economic Review 79, 957-970.

Klein, P.A., 2000. National statistics, portrait of the value floor. Journal of Economy 34, 445-452.

Kristandl, G., Bontis, N., 2007. Constructing a definition for intangibles using the resource based view of the firm. Management Decision 45, 1510-1524.

López, V.R., Nevado, D., Baños, J., 2008. Indicador sintético de capital intelectual: humano y estructural. Un factor de competitividad. EURE (Santiago) 34, 45-70.

López, V.R., Nevado, D., 2016. Strategic control model from the perspective of the value of intangibles. Method and application. Innovar 26, 9-20.

López-Nicolás, C., Meroño-Cerdán, A.L., 2011. Strategic knowledge management, innovation and performance. International Journal of Information Management 31, 502-509.

Lucas, R., 1988. On the mechanics of economic development. Journal of Monetary Economics 22, 3-42.

Malhotra, Y., 1998. Deciphering the knowledge management hype. Journal for Quality \& Participation 21, 58-60.

Malhotra, Y., 2000. Knowledge management and new organization forms: A framework for business model innovation. Information Ressources Management Journal 13, 5-14.

Marcotte, C., 2013. Measuring entrepreneurship at the country level: A review and research agenda. Entrepreneurship and Regional Development 25, 174-194.

Martín de Castro, G., Alama, E., Navas, J.E., López, P., 2009. El papel del capital intelectual en la innovación tecnológica: Una aplicación a las empresas de servicios profesionales de España. Cuadernos de Economía y Dirección de la Empresa 40, 83-110.

Masson, J.L., 2007. El capital emprendedor como determinante del crecimiento económico en España [trabajo de investigación]. UAB, Barcelona.

Matejovsky, L., Mohapatra, S., Steiner, B., 2014. The dynamic effects of entrepreneurship on regional economic growth: Evidence from Canada. Growth and Change 45, 611-639.

Müller, S., 2016. A progress review of entrepreneurship and regional development: What are the remaining gaps? European Planning Studies 24, 1133-1158.

Muñoz-Seca, B., Riverola, J., 1997. Gestión del Conocimiento. Barcelona. Biblioteca IESE de Gestión de Empresas. Universidad de Navarra, Navarra.

Nonaka, I., Takeuchi, H., 1995. The Knowlwdge Creating Company. Oxford University Press, New York.

Piergiovanni, R., Carree, M.A., Santarelli, E., 2012. Creative industries, new business formation, and regional economic growth. Small Business Economics 39, 539-560.

Porter, M.E., Millar, V., 1986. Cómo obtener ventajas competitivas por medio de la información. Harvard-Deusto Business Review 25, 3-20.

Prados, L., Rosés, J.R., 2008. Long-run estimates or physical capital in Spain, 1850-2000. Madrid. En: Working Papers in Economic History, WP 08-07. Universidad Carlos III de Madrid, Madrid.
Pulido, A., Rodríguez-Vález, J., 2006. ¿Afecta la multicolinealidad al análisis empírico de la productividad de las infraestructuras regionales? Madrid. Working Paper. Instituto Lawrence R. Klein, Universidad Autónoma de Madrid, Madrid.

Ramírez, Y., 2010. Medición y gestión del capital intelectual en el ámbito territorial. Revista de Estudios Regionales 88, 223-252.

Reynolds, P.D., 1999. Creative Destruction: Source or Symptom of Economic Growth? En: Acs, Z.J., Carlsson, B., Karlsson, C. (Eds.), Entrepreneurship, Small and Medium-Sized Enterprises and the Macroeconomy. Cambridge University Press, Cambridge.

Romer, P.M., 1986. Increasing returns and long-run growth. Journal of Political Economy 94, 1002-1037.

Romer P.M., 1990. Endogenous Technological Change. Journal of Political Economy, 98 (5), Part 2: The Problem of Development: A Conference of the Institute for the Study of Free Enterprise Systems (October), S71-S102.

Sánchez, S., Arellano, A., 2012. El empleo en la economía del conocimiento en España: características socio-profesionales y patrones de distribución territorial. Scripta Nova 16, 399.

Schumpeter, J.A., 1934. Entrepreneurship as innovation. Oxford University Press, Oxford.

Sheram, K., Soubbotina, T., 2005. Beyond Economic Growth. Meeting the Challenges of Global Development, Banco Mundial.

Simó, P., Sallán, J.M., 2008. Capital intangible y capital intelectual: Revisión, definiciones y líneas de investigación. Estudios de Economía Aplicada 26, 65-78.

Skyrme, D., 2001. Capitalizing on Knowledge: From E-Business to K-Business. Butterworth-Heinemann, Oxford.

Solow, R.M., 1956. A contribution to the theory of economic growth. The Quarterly Journal of Economics 70, 65-94.

Sternberg, R., Wennekers, S., 2005. Determinants and effects of new business creation using Global Entrepreneurship Monitor data. Small Business Economics 24, 193-203.

Stewart, T.A., 1997. Intellectual Capital: The New Wealth of Organizations. Doubleday, New York.

Veciana, J.M., 2007. Entrepreneurship as a Scientific Research Program. En: Cuervo, A., Ribeiro, D., Roig, S. (Eds.), Entrepreneurship: Concepts, Theory and Perspective. Springer, New York, pp. 23-72.

Subramaniam, M., Youndt, M.A., 2005. The influence of intellectual capital on the types of innovative capabilities. Academy of Management Journal 48, 450-463.

Sullivan, P.H., 1998. Profiting from Intellectual Capital: Extracting Value from Innovation. Wiley, New York.

Viedma, J.M., 2007. In search of an Intellectual Capital comprehensive theory. Electronic Journal of Knowledge Management 5, 245-256.

Wennekers, A.R.M., 2006. Entrepreneurship at the Country Level: Economic and Non-economic Determinants. ERIM, Rotterdam.

Wooldridge, J.M., 2002. Econometric Analysis of Cross Section and Panel Data. MIT Press, Cambridge.

Zack, M.H., 2002. Developing a Knowledge Strategy: Epilogue. En: Choo, C.W., Bontis, N. (Eds.), The Strategic Management of Intellectual Capital and Organizational Knowledge: A Collection of Readings. Oxford University Press, Oxford, pp. 268-276. 